\title{
BMJ Open Is patient participation in multidisciplinary tumour conferences associated with their information needs? A multicentre prospective observational study
}

\author{
Sebastian Reck (D) , ${ }^{1}$ Annika Diekmann, ${ }^{1}$ Christian Heuser (D) , \\ Barbara Schellenberger, ${ }^{1}$ Lena Ansmann, ${ }^{2,3}$ Markus Antonius Wirtz, ${ }^{4}$ \\ Nicole Ernstmann (i) ${ }^{1}$
}

To cite: Reck S, Diekmann A, Heuser $\mathrm{C}$, et al. Is patient participation in multidisciplinary tumour conferences associated with their information needs? A multicentre prospective observational study. BMJ Open 2021;11:e049199. doi:10.1136/ bmjopen-2021-049199

- Prepublication history for this paper is available online. To view these files, please visit the journal online (http://dx.doi org/10.1136/bmjopen-2021049199).

Received 18 January 2021 Accepted 31 August 2021
Check for updates

(C) Author(s) (or their employer(s)) 2021. Re-use permitted under CC BY-NC. No commercial re-use. See rights and permissions. Published by BMJ.

For numbered affiliations see end of article.

Correspondence to

Sebastian Reck;

Sebastian.Reck@ukbonn.de

\section{ABSTRACT}

Objectives To determine the association between participation of patients with breast cancer and patients with gynaecological cancer in their own multidisciplinary tumour conference (MTC) and their information needs with regard to their disease and treatment options.

Methods This is a prospective observational study that took place at six breast cancer and gynaecological cancer centres in North Rhine-Westphalia, Germany. Patient inclusion criteria included a minimum age of 18 years and at least one diagnosis of breast cancer or gynaecological cancer. Three surveys were administered. T0 (shortly before MTC), T1 (directly after MTC) and T2 (4 weeks after MTC). Patient information needs were measured using two three-item subscales of the Cancer Patients Information Needs scale. Analysis of change was done by one-way repeated measures analysis of variance (ANOVA). To control for sampling bias, a further one-way repeated measures analysis of covariance (ANCOVA) included a propensity score as a covariate.

Results Data from 81 patients in the participation group and 120 patients in the non-participation group were analysed. The patient groups did not differ in their levels of information needs at T0 or T2. From T0 to T2, information needs increased statistically significantly in both groups with regard to both disease-related information $\left(\eta^{2}=0.354\right)$ and treatment-related information $\left(\eta^{2}=0.250\right)$. The increase in both types of information needs lost its statistical significance when the propensity score was included as a covariate. Neither ANOVA nor ANCOVA revealed a statistically significant association between patients' participation in the MTC and their self-reported information needs.

Conclusion and clinical implications As concerns patients' information needs, findings do not support a general recommendation for or against the participation of patients in their MTCs. Future research should focus on the different ways of patients' participation in their MTCs facilitated at different cancer centres. Further research should also aim to establish which patient and disease characteristics predispose patients to benefit from participating in their MTCs.
Strengths and limitations of this study

Drawing on a multicentre sample, this is the first study to quantitatively assess the association between patients' participation in their multidisciplinary tumour conference (MTC) and their information needs.

- A wide spectrum of sociodemographic, disease and psychosocial confounder variables were surveyed at multiple points in time.

- Using abovementioned confounders, a propensity score was calculated in order to further justify the comparison of patients participating in their MTCs with non-participants in this non-experimental setting.

- Given the overall sample size of 201 patients, the number of patients in some cancer centres was small.

During the research, it became clear that there is variation in the way patients' participation in their MTCs takes place at different cancer centres.

\section{INTRODUCTION}

The importance of meeting the information needs of patients with cancer has been established by a large and growing body of literature. This holds true for both diseaserelated and treatment-related information. The provision of adequate information helps patients with cancer to reduce uncertainty and regain a sense of control, thus enabling them to make informed decisions with regard to their treatment. ${ }^{12}$ It is furthermore associated with higher patient satisfaction and increased compliance with treatment programmes. ${ }^{3}$ Meeting patients' information needs can be associated with improved health outcomes such as decreased levels of anxiety and depression and improvements in the ability to cope. ${ }^{4-6}$ 
The information needs of patients with cancer are generally high. ${ }^{25}$ Factors such as female sex, younger age, lower level of education, living with a partner, a short time since diagnosis and different tumour sites have been linked to particularly high information needs. ${ }^{2} 4$ It has been shown that patients are primarily interested in information on the nature and stage of their disease, treatment options and potential adverse effects of treatment. ${ }^{27}$ These information needs are not static but vary over the course of the disease/treatment. ${ }^{5}$

Despite these insights, a mismatch between patients' information needs and the actual supply of information prevails. The information needs of many patients with cancer remain unmet. ${ }^{2}$ This holds especially true for information on medical examination results, treatment options, side effects of medication ${ }^{5}$ and psychological support. ${ }^{28}$ Patients with lower levels of health literacy seem to be at a particular disadvantage. ${ }^{5}$ They appear to have trouble identifying their own information needs, understanding medical information and even communicating their lack of understanding. ${ }^{4}$

As a potential remedy to this problem, it was found that a close and trustful patient-clinician relationship predicts lower levels of unmet information needs. ${ }^{4}$ For most patients, their attending physicians and nurses are the most trusted sources of information. ${ }^{6}$ It is therefore conceivable that patients' participation in their own multidisciplinary tumour conferences (MTCs) may contribute to the satisfaction of their information needs. After all, the MTC is precisely the setting in which the information most relevant to patients (namely diagnosis and treatment options) is discussed face to face by (or with) all specialists involved.

In the context of the growing complexity of oncological treatments, MTCs have become a central instrument of multidisciplinary oncological care. ${ }^{9-11}$ Their main purpose is to facilitate cooperation between medical professionals by providing a forum to discuss individual oncological cases and to jointly consider the best treatment options. ${ }^{911}$

In Germany, MTCs form part of the clinical practice guideline for the treatment of breast cancer as defined by the German Cancer Society, German Cancer Aid and the Association of the Scientific Medical Societies of Germany. ${ }^{12}$ Moreover, in the wake of a broader trend towards patient-centred care, breast care centre certification criteria in North Rhine-Westphalia, Germany's most populous federal state, grant every patient the right to attend and participate in the MTC discussing their case. ${ }^{13}$

So far, this is not common practice in German oncological healthcare. Only a minority of patients are invited to attend their MTCs. Nevertheless, of those invited, at least half $^{1415}$ attend, and the vast majority of attendees does not regret doing so. ${ }^{15}{ }^{16} \mathrm{Up}$ until now, international research on patients' participation in their MTCs has been mainly exploratory. In an Australian pilot study of 2007, Choy et al assessed the feasibility of including patients with breast cancer in their MTCs. The initiative was highly valued by the majority of patients and at least deemed acceptable by most medical service providers involved. Recent research from Germany focused on characteristics predisposing patients for participation as well as patients' perception of this type of initiative. ${ }^{141517}$ It was found that inadequate levels of health literacy are associated with a lower likelihood of participation. ${ }^{17}$ Furthermore, patients with a higher educational level were more likely to accept the invitation to their MTCs. ${ }^{14}$ When asked to describe their experience, most MTC participants perceived it to be supportive and informative. ${ }^{15}$

On the other hand, MTCs are often very fast-paced technical meetings in which information that is potentially life changing for individual patients is encoded in specialised medical terms and abbreviations. ${ }^{18}$ Rather than satisfying patients' information needs, such a setting can run the risk of overburdening patients both cognitively and emotionally. This may result in the opposite of the intended effect, namely confusion, and even an increase in anxiety. ${ }^{16151920}$

The aim of this study is to find out if participation in an MTC is associated with the information needs of patients with breast cancer and patients with gynaecological cancer. It specifically addresses the following questions: (a) Do participating patients differ in their information needs as compared with patients who do not participate in their MTCs? (b) How does the need for information change in these groups over the course of 4 weeks? (c) Are changes in the need for information associated with MTC participation versus non-participation?

\section{METHODS}

\section{Study design and sample}

This analysis forms part of a larger research project named 'Patient participation in multidisciplinary tumor conferences in breast cancer care' (PINTU). PINTU is a multicentre observational mixed-methods study. Further details on the PINTU study design are reported in the study protocol. ${ }^{21}$ The project was approved by the ethics committee of the Faculty of Medicine of the University of Cologne, Germany, and received funding from the German Cancer Aid. Data collection took place from November 2018 until February 2020 at six breast cancer and gynaecological cancer centres in North RhineWestphalia. Cancer centres were chosen based on case volume treated and teaching status. ${ }^{21}$ Three out of six centres offered patients the possibility to participate in their MTCs.

Patient inclusion criteria were a minimum age of 18 years, at least one diagnosis of breast cancer or gynaecological cancer (C50.xx-C58.xx, D05.xx-D07.xx), the psychological and cognitive ability to give written consent and fill out questionnaires, alongside sufficient German language skills. Both participation group (PG) and non-participation group (N-PG) respondents were recruited by different healthcare providers in each clinic. Patients' informed consent to participate was obtained 


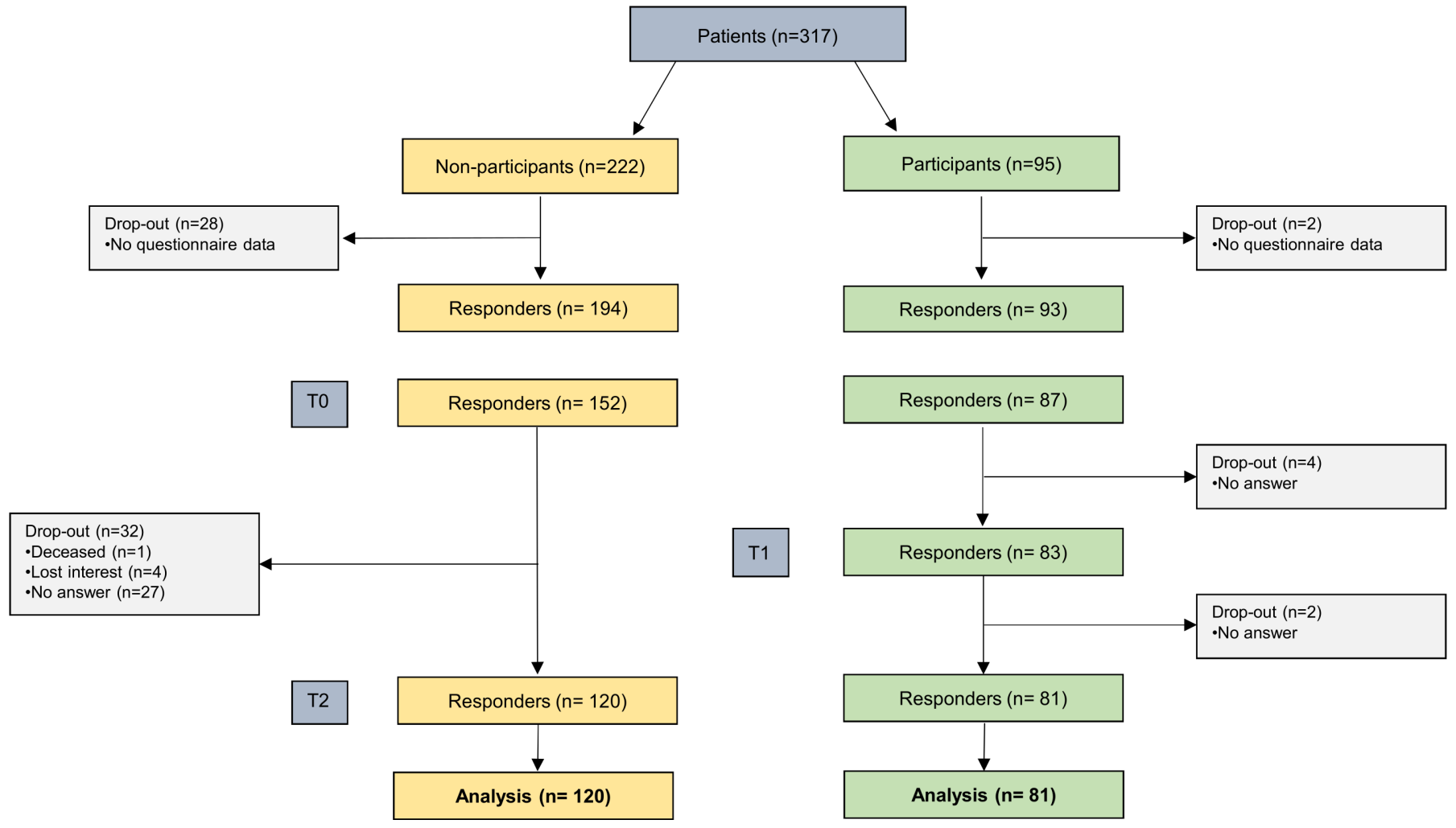

Figure 1 Flow of participants.

in individual conversations and documented in written form. They completed a total of three questionnaires at different points in time. T0 was shortly before the MTC, T1 right after attending their MTCs and T2 4 weeks after T0. N-PG members completed questionnaires at T0 and T2. For both PG and N-PG, the present analysis focuses exclusively on T0 and T2. Applying a modified version of the Dillman Total Design Method, ${ }^{22}$ T0 questionnaires were handed out by clinic staff, T1 by members of the research team and T2 questionnaires reached participants via postal service. A total of 201 respondents (81 PG and $120 \mathrm{~N}-\mathrm{PG}$ ) were included in the analysis. The flow of participants and non-responders' reasons are listed in figure 1 .

\section{Instruments}

Patients' information needs were measured using an adapted version of the Cancer Patients Information Needs Scale (CaPIN) developed by Neumann et al. ${ }^{4}$

Six items were used to assess patients' information needs at both T0 and T2. Each item was introduced by the question 'would you have liked more information about xxx' and had to be answered on a dichotomous answer scale (yes/no).

For the purpose of analysis, these six items were further subdivided into two subscores consisting of three items each, seeking to assess patients' information needs pertaining to

- The disease itself (CaPIN_disease; including examination results, diagnosis, course of disease; $\alpha=0.897$ ) and
- The treatment (CaPIN_treatment; including advantages/disadvantages of treatment, medication, means to reduce the adverse effects of medication; $\alpha=0.882$ ).

If a patient negated all three items of a subscore, this would indicate that there is currently no further need for information. A score of 3 would indicate a maximum need for (additional) information at this point in time.

Potential confounder variables with regard to patients' information needs were derived deductively from the literature ${ }^{23-27}$ and included in the questionnaire administered at T0. Sociodemographic characteristics included age, level of education, health literacy ${ }^{26}$ (16-item scale; $\alpha=0.85$ ), living with a partner, marital status, employment status, having children and type of health insurance. The T0 questionnaire also surveyed psychosocial confounder variables such as need for participation ${ }^{27}$ (6-item scale; $\alpha=0.758$ ), need for informational education ${ }^{27}$ (4-item scale; $\alpha=0.811$ ), paternalism preference ${ }^{27}$ (4-item scale; $\alpha=0.800$ ), self-help preference ${ }^{27}$ (4-item scale; $\alpha=0.653$ ), need for psychosocial support, ${ }^{23}$ distress thermometer, ${ }^{25}$ fear of cancer progression ${ }^{28}$ (12-item scale; $\alpha=0.849$ ) and therapy confidence. ${ }^{23}$ Clinical information comprised size of tumour, lymph node status, possible metastasis and histological characteristics of the malignancy as summarised by the Union for International Cancer Control staging system. ${ }^{24}$ This information was obtained predominantly from the audio recordings of the MTCs or from the medical service providers. The cancer centres were coded from 1 to 6 . 


\section{Analysis}

Only respondents who had filled out both questionnaires T0 and T2 were included in the analysis. To avoid a decrease in sample size as well as bias due to data missing not at random, missing values were imputed using the expectation-maximisation algorithm implemented in the software NORM. This method is most appropriate if missing values are not completely missing at random, meaning that they can be estimated by using other variables in the dataset. ${ }^{29}$ For categorical covariates, missing values were substituted with the mode value. Overall, missings per item did not exceed $21 \%$.

Data was analysed using IBM SPSS Statistics V.26. In order to establish T0 baseline differences between PG and N-PG, t-tests, $\chi^{2}$-tests and Mann-Whitney $U$ tests were calculated (see table 1). This analysis included the abovementioned sociodemographic, psychosocial and clinical confounder variables.

Given the fact that study participants were not assigned randomly to PG and N-PG, a propensity score ${ }^{30}$ (including all of the variables in table 1 ) was calculated in order to mitigate the problems of sampling bias by the medical staff and self-selection of patients into the PG or N-PG. The propensity score is determined by a multiple logistic regression. It represents an approximation of a patient's probability to participate in an MTC.

The main analysis, seeking to establish the potential association between patients' participation and their information needs, was done by analysis of variance (ANOVA) with repeated measures. The two-stage withinsubject factor was patients' information needs at T0 and T2. The between-subject factor was participation/ non-participation in the MTC (PG vs N-PG). In order to control for potential confounders, an analysis of covariance (ANCOVA) was calculated, including the propensity score as a covariate.

\section{Patient and public involvement}

In order to ensure that the research focuses on issues relevant to patients and the public, patients were included right from the beginning of the planning phase. Two representatives of Frauenselbsthilfe Krebs (a German self-help network for women suffering from cancer) pretested the questionnaires. Most of the outcome measures had been drafted based on prior research by the University of Cologne. ${ }^{23}$ Patient representatives were not reimbursed financially. On first contact with the researchers, patients were informed about the time required to participate in this study (both verbally and in written). Ward physicians and nurses informed participants about the potential advantages and disadvantages of participating in the MTC. Results so far were shared with participating breast care centres and patient representatives in a closing workshop.

\section{RESULTS}

\section{Baseline comparison of PG and N-PG}

A total of $n=317$ patients were recruited for the study. A total of 201 patients (81 participants (PG) and 120 non-participants (N-PG)) completed questionnaires at both T0 and T2 and were included in the present analysis. Table 1 displays the socioeconomic, psychosocial and clinical variables in both PG and N-PG at T0 (shortly before MTC). In general, PG and N-PG proved to be very similar with regard to these characteristics. On average, PG patients were approximately 2years younger and showed a higher rate of stage IV cancer. However, none of these differences proved to be statistically significant.

\section{Changes in disease-related information needs from T0 to T2}

Table 2 shows the changes in disease-related information needs in both groups (PG and N-PG) from T0 to T2. The first row displays the results of the ANOVA and the second row those of the ANCOVA (with the propensity score as a covariate).$^{31}$ Neither at T0 $(\mathrm{t}(199)=0.394$, $\mathrm{p}>0.05)$ nor at $\mathrm{T} 2(\mathrm{t}(199)=0.496, \mathrm{p}>0.05)$ were there statistically significant differences between the PG and the N-PG with regard to disease-related information (CaPIN_ disease). Starting from a moderate level, it increased more than one scale point from $\mathrm{T} 0$ to $\mathrm{T} 2(\mathrm{~F}=108.873$; $\left.\mathrm{df}=1 ; \mathrm{p}=<0.001 \uparrow ; \eta^{2}=0.354\right)$. However, participation in the MTC showed no effect on CaPIN_disease $(\mathrm{F}=0.000 ; \mathrm{df}=1$; $\left.\mathrm{p}=0.987 ; \eta^{2}=<0.001\right)$. Controlling for propensity score as a covariate did not significantly explain the variance in the change of CaPIN_disease $(\mathrm{F}=2.406 ; \mathrm{df}=1 ; \mathrm{p}=0.122$; $\left.\eta^{2}=0.012\right)$. It does, however, make the main effect of time lose its statistical significance $(\mathrm{F}=2.028 ; \mathrm{df}=1 ; \mathrm{p}=0.156$; $\left.\eta^{2}=0.01\right)$. Figure 2 is a graphical representation of the changes in CaPIN_disease over time.

Changes in treatment-related information needs from T0 to T2 Table 3 shows the changes in treatment-related information needs (CaPIN_treatment) in both groups (PG and N-PG) from T0 to T2. The first row displays the results of the ANOVA and the second row those of the ANCOVA (with the propensity score as a covariate). ${ }^{31}$ Neither at T0 $(\mathrm{t}(199)=0.508, \mathrm{p}>0.05)$ nor at T2 $(\mathrm{t}(199)=-0.296, \mathrm{p}>0.05)$ were there statistically significant differences between the PG and the N-PG with regard to treatment-related information needs (CaPIN_treatment). Starting from a moderate level, CaPIN_treatment significantly increased in both groups from T0 to T2 $(\mathrm{F}=66.431$; $\mathrm{df}=1 ; \mathrm{p}<0.001 \uparrow$; $\left.\eta^{2}=0.250\right)$. However, participation in the MTC showed no effect on CaPIN_treatment $(\mathrm{F}=0.525$; df $=1 ; \mathrm{p}=0.47$; $\left.\eta^{2}=0.003\right)$. Including the propensity score as a covariate does not add to explaining variance in CaPIN_treatment $\left(\mathrm{F}=2.288 ; \mathrm{df}=1 ; \mathrm{p}=0.132 ; \eta^{2}=0.011\right)$. It does, however, make the main effect of time lose its statistical significance $\left(\mathrm{F}=0.677 ; \mathrm{df}=1 ; \mathrm{p}=0.412 ; \eta^{2}=0.003\right)$. Figure 3 is a graphical representation of the changes in CaPIN_treatment over time.

\section{DISCUSSION}

In an observational study that took place at six breast cancer and gynaecological cancer centres in North RhineWestphalia, Germany, we investigated the association 
Table 1 Sample characteristics by participation group and non-participation group at baseline (TO)

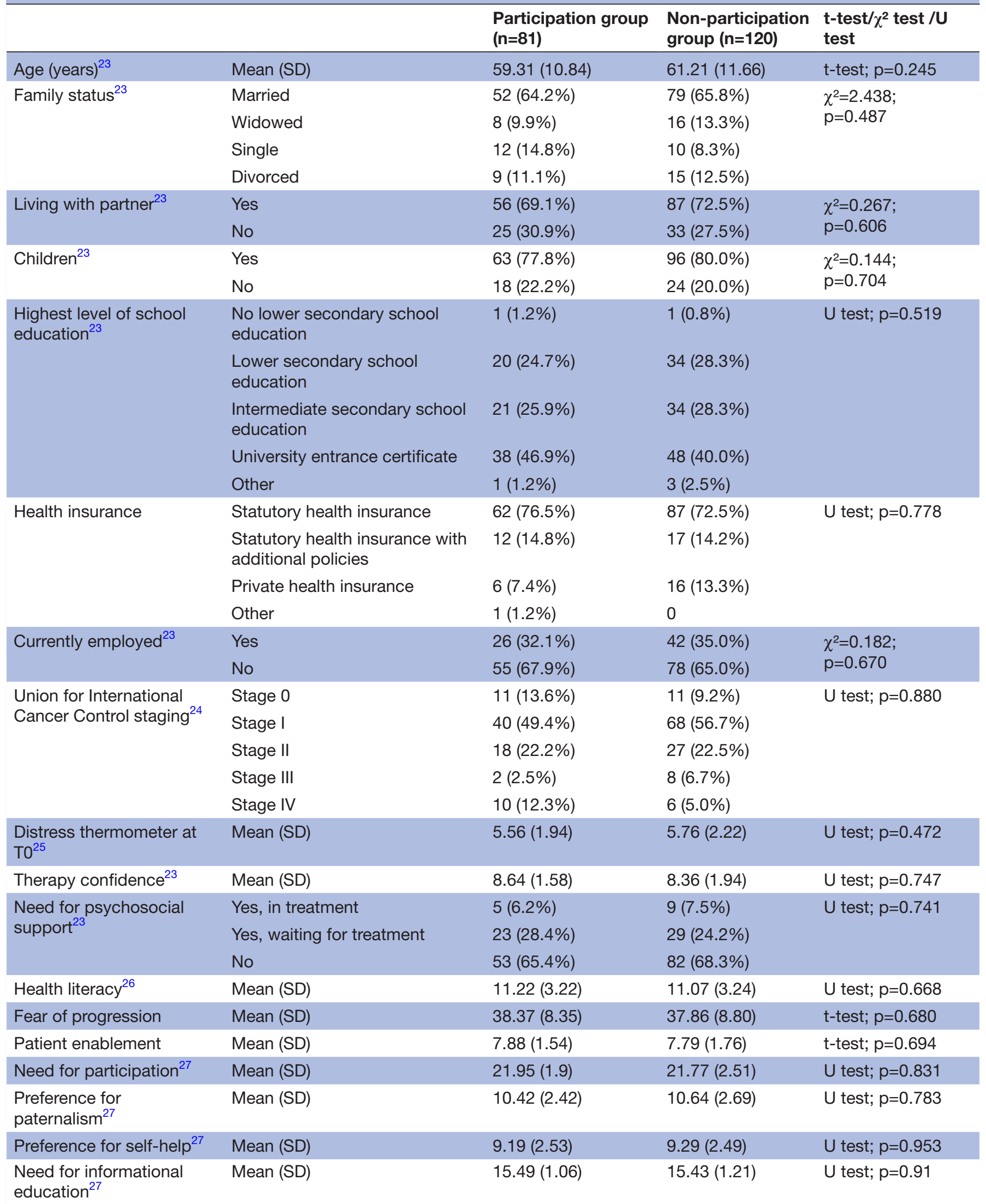

*Statistically significant at $5 \%$ level.

†Statistically significant at $1 \%$ level.

p, probability; T0, first point of measurement; T2, third point of measurement; $U$ test, Mann-Whitney $U$ test. 
Table 2 Changes in CaPIN_disease scores from before MTC (T0) to 4 weeks after MTC (T2), broken down by participation group ( $n=81)$ and non-participation group $(n=120)$. First row ANOVA, second row ANCOVA controlled for propensity score. Columns T0 and T2 are descriptive statistics of participation group and non-participation group and apply to both analyses

\begin{tabular}{|c|c|c|c|c|c|c|c|c|c|c|c|c|c|}
\hline & \multicolumn{2}{|l|}{ T0 } & \multicolumn{2}{|l|}{ T2 } & \multicolumn{3}{|c|}{ Main effect time } & \multicolumn{3}{|c|}{$\begin{array}{l}\text { Interaction } \\
\text { (time*propensity } \\
\text { score) }\end{array}$} & \multicolumn{3}{|c|}{$\begin{array}{l}\text { Interaction } \\
\text { (time*group) }\end{array}$} \\
\hline & Mean & SD & Mean & SD & $F_{\mathrm{df1}}$ & $\mathbf{p}$ & $P, \eta^{2}$ & $\mathbf{F}_{\mathrm{df1}, \mathrm{df} 2}$ & $\mathbf{p}$ & $P, \eta^{2}$ & $\mathbf{F}_{\mathrm{df1}}$ & p & $P, \eta^{2}$ \\
\hline Participation group & 1.259 & 1.302 & 2.321 & 1.082 & 108.873 & $<0.001 \dagger$ & 0.354 & - & - & - & 0.000 & 0.987 & $<0.001$ \\
\hline $\begin{array}{l}\text { Non-participation } \\
\text { group }\end{array}$ & 1.183 & 1.366 & 2.242 & 1.130 & 2.028 & 0.156 & 0.01 & 2.406 & 0.122 & 0.012 & 0.296 & 0.587 & 0.001 \\
\hline
\end{tabular}

Interpretation of effect size (ES): partial eta-squared $\left(\eta^{2}\right) \geq 0.0099=$ small ES, partial $\left(\eta^{2}\right) \geq 0.0588=$ medium ES and partial $\left(\eta^{2}\right) \geq 0.1379=\operatorname{large}$ ES. ${ }^{30}$ ${ }^{*}$ Statistically significant at $5 \%$ level. †Statistically significant at $1 \%$ level.

ANCOVA, Analysis of covariance; ANOVA, analysis of variance; CaPIN_disease, patients with cancer disease related information needs; F, test statistic; MTC, multidisciplinary tumour conference; $\mathrm{p}$, probability; T0, first point of measurement; T2, third point of measurement.

between patients' participation in their MTCs and the changes in their information needs.

It was found that PG and N-PG patients did not differ significantly in their levels of information needs at either point in time (T0, T2). With participating and nonparticipating patients displaying similar levels of education, family status and age structure, among many other psychosocial and clinical traits, the observed similarity in information needs is the expected outcome. This affirms the findings of prior studies identifying variables predictive for increased information needs. ${ }^{25}$

The analysis revealed a statistically significant increase in information needs pertaining to both the disease and the treatment options in both the PG and the N-PG $(\mathrm{T} 0 \rightarrow \mathrm{T} 2)$. This is in line with Halbach et a $\bar{l}^{\check{b}}$ who found that the need of patients with breast cancer for this information increased within the first 10 weeks after surgery.
Interestingly, when controlling the analysis for the variables contained in the propensity score, this increase loses its statistical significance for both types of information needs (disease-related and treatment-related). This is in line with a large body of research that has found associations between patients' sociodemographic, psychosocial and disease attributes on the one hand and their information needs on the other. ${ }^{1-3} 1732$ It seems that these variables explain a relevant (although statistically not significant) part of the variation in patients' information needs from $\mathrm{T} 0$ to $\mathrm{T} 2$.

The main aim of this analysis was to find out if participation in an MTC is associated with the information needs of patients with breast cancer and patients with gynaecological cancer. Its central finding is that participation in the MTC did not have any statistically significant effect on patients' information needs. This affirms the findings of

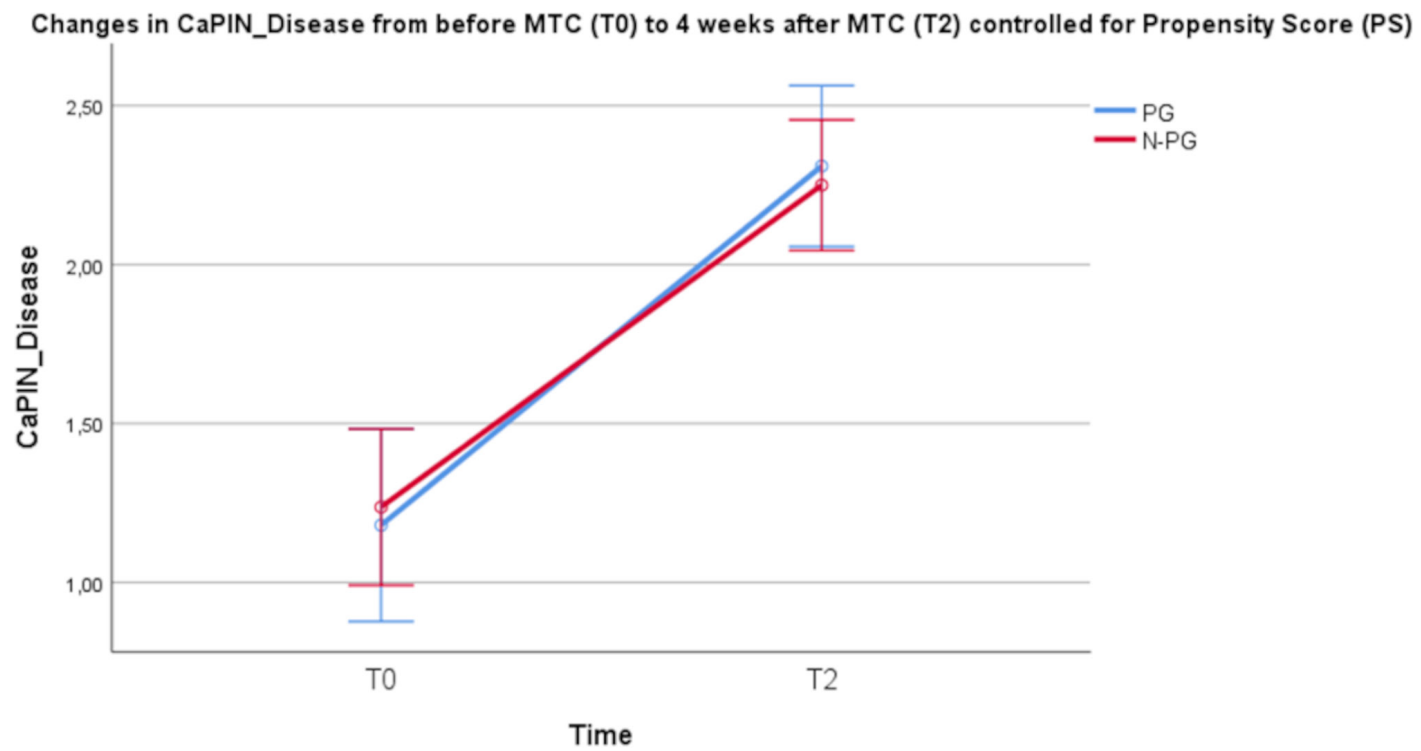

Error bars: $95 \% \mathrm{Cl}$

Figure 2 Changes in CaPIN_Disease. CaPIN, Cancer Patients Information Needs; MTC, multidisciplinary tumour conference; N-PG, non-participation group; PG, participation group 
Table 3 Changes in CaPIN_treatment scores from before MTC (T0) to 4 weeks after MTC (T2), broken down by participation group ( $n=81)$ and non-participation group $(n=120)$. First row ANOVA, second row ANCOVA controlled for propensity score. Columns T0 and T2 are descriptive statistics of participation group and non-participation groups and apply to both analyses

\begin{tabular}{|c|c|c|c|c|c|c|c|c|c|c|c|c|c|}
\hline & \multirow{2}{*}{$\begin{array}{l}\text { T0 } \\
\text { Mean }\end{array}$} & \multicolumn{3}{|c|}{ T2 } & \multicolumn{3}{|c|}{ Main effect time } & \multicolumn{3}{|c|}{$\begin{array}{l}\text { Interaction (time* } \\
\text { propensity score) }\end{array}$} & \multicolumn{3}{|c|}{$\begin{array}{l}\text { Interaction } \\
\text { (time* group) }\end{array}$} \\
\hline & & SD & Mean & SD & $\mathbf{F}_{\mathrm{df1}, \mathrm{df} 2}$ & $\mathbf{p}$ & $P, \eta^{2}$ & $\mathbf{F}_{\mathrm{df1}, \mathrm{df} 2}$ & $\mathbf{p}$ & $P, \eta^{2}$ & $\mathbf{F}_{\mathrm{df1}, \mathrm{df} 2}$ & $\mathbf{p}$ & $P, \eta^{2}$ \\
\hline $\begin{array}{l}\text { Non-participation } \\
\text { group }\end{array}$ & 1.017 & 1.303 & 1.917 & 1.234 & 0.677 & 0.412 & 0.003 & 2.288 & 0.132 & 0.011 & 0.028 & 0.866 & 0.000 \\
\hline
\end{tabular}

Interpretation of effect size (ES): partial eta-squared $\left(\eta^{2}\right) \geq 0.0099=$ small ES, partial $\left(\eta^{2}\right) \geq 0.0588=$ medium ES and partial $\left(\eta^{2}\right) \geq 0.1379=$ large ES. ${ }^{31}$

*Statistically significant at $5 \%$ level.

†Statistically significant at $1 \%$ level.

ANCOVA, analysis of covariance; ANOVA, analysis of variance; CaPIN_treatment, patients with cancer treatment related information needs; F, test statistic; Mean, mean value; MTC, multidisciplinary tumour conference; p, probability; T0, first point of measurement; T2, third point of measurement.

a more explorative 2007 study of Choy et al. ${ }^{16}$ However, it is slightly at odds with the results of a more recent qualitative study by Diekmann et $a l^{15}$ in which patients had frequently mentioned 'being better informed about their illness' as a positive outcome of participation in their MTCs. A possible explanation for this discrepancy may be the use of different instruments (two closed-question, 3-item scales vs open question).

The absence of a statistically significant effect may be owed to patient participation in MTC being a variation of-rather than an alternative to-the health service of information. PG patients did not necessarily receive more information than N-PG patients. Rather, it was the delivery of this information that differed between groups.

\section{Strengths and limitations}

The strengths and limitations of our study are closely intertwined. The fact that it took place at six cancer centres in North Rhine-Westphalia gives it a broader scope than previous studies. However, not all centres offered the possibility of MTC participation, meaning that some of them contributed exclusively to the N-PG.

Moreover, this being a multicentre study meant that, for logistical reasons, the sampling of both PG and N-PG participants was done mainly by local medical staff. Previous research indicates that this process likely does not occur in a randomised manner. Rather, beyond inclusion and exclusion criteria, who is invited to the MTC partly depends on patients' sociodemographic, disease

\section{Changes in CaPIN_Treatment from before MTC (T0) to 4 weeks after MTC (T2) controlled for Propensity Score (PS)}

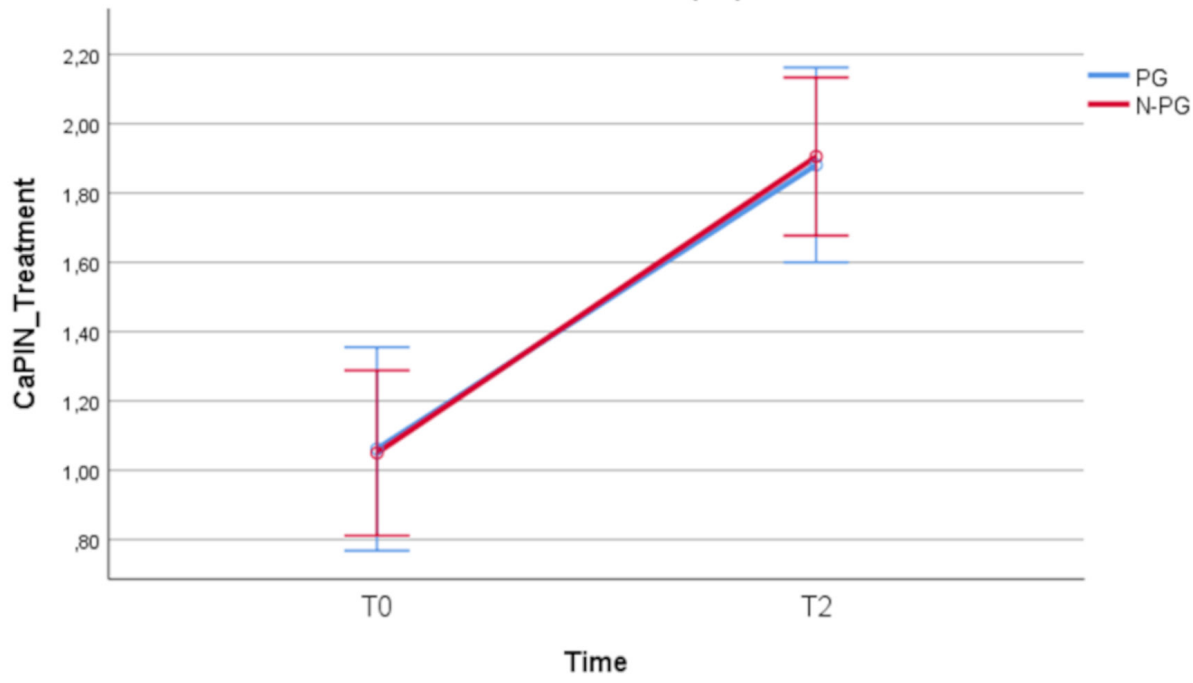

Error bars: $95 \% \mathrm{Cl}$

Figure 3 Changes in CaPIN_Treatment. CaPIN, Cancer Patients Information Needs; MTC, multidisciplinary tumour conference; N-PG, non-participation group; PG, participation group. 
and treatment characteristics. Self-selection effects based on patients' health literacy ${ }^{17}$ and level of education ${ }^{14}$ also needed to be taken into account.

The T0 baseline comparison revealed no statistically significant differences between PG and N-PG with regard to relevant confounder variables. Nevertheless, to further justify the comparison of PG and N-PG, we constructed a comprehensive propensity score, using it as a covariate in the main analysis. While this is a strength of our study, it is important to keep in mind that the propensity score may have missed some unknown but relevant confounder variables.

Another limitation is the relatively short period of time, mostly just over 4 weeks, that lies between $\mathrm{T} 0$ and T2. While this was necessary to detect immediate effects of the MTC, it may have been insightful to include a later point of measurement in order to depict changes in patients' demand for different types of information. Furthermore, the period of measurement was not strictly standardised to the patients' course of disease. Patients answered T0 mostly very briefly before the MTC, which for most patients means at a time point shortly after diagnosis, but not necessarily at precisely the same point of their 'cancer journey'.

Finally, conducting the study at six different cancer centres revealed that the intervention of patients participating in their own MTCs was not implemented homogenously. Rather, clinics had found different ways to offer this opportunity to their patients. A more qualitative analysis, which is also part of the PINTU research project, will shed more light on this.

\section{Implications and further research}

From a patient risk and benefits perspective, our findings do not support a general recommendation for or against the participation of patients in their MTCs. However, bearing in mind the risk of cognitive and emotional overload, it is important that medical staff screen and adequately brief patients as to what they may expect from participation. Future research should focus on the different ways of patients' participation in their MTCs facilitated at different cancer centres. It seems likely that the different organisational approaches and communication styles may directly influence patients' satisfaction with the information provided. Further research should also aim to establish which types of patient and disease characteristics predispose patients to benefit from participating in their MTCs.

\section{CONCLUSION}

Patients with breast cancer and patients with gynaecological cancer have unmet information needs in terms of diagnosis and treatment. The present study did not find any statistically significant effect of patients' participation in their MTCs on their information needs with regard to the disease itself and its treatment. Participants showed similar levels of information needs over time. Both disease-related and treatment-related information needs increased significantly from T0 to T2. This statistical significance is lost when controlling for the propensity score, implying that the sociodemographic, psychosocial and disease-related variables contained in the score explain parts of the variance in information needs.

\section{Author affiliations}

${ }^{1}$ Center for Health Communication and Health Services Research, Department for Psychosomatic Medicine and Psychotherapy, University Hospital Bonn, Bonn, Germany

${ }^{2}$ Department of Organizational Health Services Research, University of Oldenburg, Oldenburg, Germany

${ }^{3}$ Faculty of Human Sciences and Faculty of Medicine, Institute of Medical Sociology, Health Services Research and Rehabilitation Science (IMVR), University of Cologne, Cologne, Germany

${ }^{4}$ Department of Research Methods, University of Education Freiburg, Freiburg im Breisgau, Germany

Contributors SR planned and conducted data analyses, drafted and revised all sections of the paper and is guarantor. AD, CH, BS, MAW, LA and NE assisted in data analysis, interpretation of the results and editing of the manuscript. MAW consulted on the statistical methods used. LA, NE, CH and AD developed the PINTU study framework. All authors read and approved the final manuscript.

Funding This work was supported by the German Cancer Aid (DKH, grant number 70112286).

Competing interests None declared.

Patient and public involvement Patients and/or the public were involved in the design, or conduct, or reporting, or dissemination plans of this research. Refer to the Methods section for further details.

Patient consent for publication Not applicable.

Ethics approval Ethics Committee of the Medical Faculty of the University of Cologne, Germany. ID: 17-405.

Provenance and peer review Not commissioned; externally peer reviewed.

Data availability statement Data may be obtained from a third party and are not publicly available. Data cannot be accessed in a repository yet. Data and all other materials for this study are kept at the Centre for Health Communication and Health Services Research (CHSR), University Hospital Bonn, Germany. The data sets generated and analysed during the current study are not publicly available due to the terms of written informed consent to which the participants agreed.

Open access This is an open access article distributed in accordance with the Creative Commons Attribution Non Commercial (CC BY-NC 4.0) license, which permits others to distribute, remix, adapt, build upon this work non-commercially, and license their derivative works on different terms, provided the original work is properly cited, appropriate credit is given, any changes made indicated, and the use is non-commercial. See: http://creativecommons.org/licenses/by-nc/4.0/.

ORCID iDs

Sebastian Reck http://orcid.org/0000-0002-3022-7009

Christian Heuser http://orcid.org/0000-0001-9050-5800

Nicole Ernstmann http://orcid.org/0000-0001-7685-6110

\section{REFERENCES}

1 Blödt S, Kaiser M, Adam Y, et al. Understanding the role of health information in patients' experiences: secondary analysis of qualitative narrative interviews with people diagnosed with cancer in Germany. BMJ Open 2018;8:e019576.

2 Faller H, Koch U, Brähler E, et al. Satisfaction with information and unmet information needs in men and women with cancer. J Cancer Surviv 2016;10:62-70.

3 Gaston CM, Mitchell G. Information giving and decision-making in patients with advanced cancer: a systematic review. Soc Sci Med 2005;61:2252-64.

4 Neumann M, Wirtz M, Ernstmann N, et al. Identifying and predicting subgroups of information needs among cancer patients: an 
initial study using latent class analysis. Support Care Cancer 2011;19:1197-209.

5 Halbach SM, Ernstmann N, Kowalski C, et al. Unmet information needs and limited health literacy in newly diagnosed breast cancer patients over the course of cancer treatment. Patient Educ Couns 2016;99:1511-8.

6 Germeni E, Bianchi M, Valcarenghi D, et al. Longitudinal qualitative exploration of cancer information-seeking experiences across the disease trajectory: the INFO-SEEK protocol. BMJ Open 2015;5:e008933.

7 Wallberg B, Michelson H, Nystedt M, et al. Information needs and preferences for participation in treatment decisions among Swedish breast cancer patients. Acta Oncol 2000;39:467-76.

8 Schmitz C, Ansmann L, Ernstmann N. Psychosocial information requirements for Multimorbid breast cancer patients in breast centres in North Rhine Westphalia. Geburtshilfe Frauenheilkd 2015;75:702-9.

9 Prades J, Remue E, van Hoof E, et al. Is it worth reorganising cancer services on the basis of multidisciplinary teams (MDTs)? A systematic review of the objectives and organisation of MDTs and their impact on patient outcomes. Health Policy 2015;119:464-74.

10 Hahlweg P, Didi S, Kriston L, et al. Process quality of decisionmaking in multidisciplinary cancer team meetings: a structured observational study. BMC Cancer 2017;17:772.

11 Wright FC, De Vito C, Langer B, et al. Multidisciplinary cancer conferences: a systematic review and development of practice standards. Eur J Cancer 2007;43:1002-10.

12 AWMF, DKG, Deutsche Krebshilfe. S3-Leitlinie mammakarzinom version 4.32020 [S3-clinical practice guideline for the treatment of breast cancer].

13 Ärztekammer Westfalen-Lippe. Verfahren zur Zertifizierung von Brustzentren in NRW 2020 [Procedure for the certification of breast care centers in North Rhine-Westphalia]. Available: https://www. aekwl.de/fileadmin/user_upload/aekwl/aekzert/Anforderungskatalog fuer_Brustzentren_NRW.pdf

14 Ansmann L, Kowalski C, Pfaff H, et al. Patient participation in multidisciplinary tumor conferences. Breast 2014;23:865-9.

15 Diekmann A, Heuser C, Ernstmann N, et al. How do breast cancer patients experience multidisciplinary tumor conferences? - A description from the patient perspective. Breast 2019;44:66-72.

16 Choy ET, Chiu A, Butow P, et al. A pilot study to evaluate the impact of involving breast cancer patients in the multidisciplinary discussion of their disease and treatment plan. Breast 2007;16:178-89.

17 Heuser C, Diekmann A, Kowalski C, et al. Health literacy and patient participation in multidisciplinary tumor conferences in breast cancer care: a multilevel modeling approach. BMC Cancer 2019;19:330.

18 Soukup T, Murtagh GM, Lamb BW, et al. Gaps and overlaps in cancer multidisciplinary team communication: analysis of speech. Small Group Res 2021;52:189-219.
19 Butow P, Harrison JD, Choy ET, et al. Health professional and consumer views on involving breast cancer patients in the multidisciplinary discussion of their disease and treatment plan. Cancer 2007;110:1937-44.

20 Diekmann A, Heuser C, Schellenberger B, et al. Patient participation in multidisciplinary tumor conferences: providers' perceptions of patients' need satisfaction and emotional experiences. Psychooncology 2020;29:1263-71.

21 Heuser C, Diekmann A, Ernstmann N, et al. Patient participation in multidisciplinary tumour conferences in breast cancer care (PINTU): a mixed-methods study protocol. BMJ Open 2019;9:e024621.

22 Dillmann DA. Mail and telephone surveys: the total design method. New York: Wiley, 1978.

23 Ansmann L, Hower K, Pfaff H. Kölner Patientenfragebogen für Brustkrebs 2.0 (KPF-BK 2.0) Kennzahlenhandbuch [Cologne patient questionnaire for breast cancer 2.0]. Köln 2015.

24 UICC Global Cancer Control. How to use TNM. Available: https:// www.uicc.org/resources/tnm [Accessed 08Oct 2020].

25 Pfaff H, Ernstmann N, Groß SE. Fragebogen für Patienten mit Kolorektalkarzinom in ambulanter onkologischer Versorgung (WIN ON Pat) Kennzahlenhandbuch [Questionnaire for outpatients with colorectal carcinoma], 2014. Available: https://docplayer.org/ 11371759-Fragebogen-fuer-patienten-mit-kolorektalkarzinomin-ambulanter-onkologischer-versorgung-win-on-patkennzahlenhandbuch.html [Accessed 08 Oct 2020].

26 Sørensen K, Van den Broucke S, Fullam J, et al. Health literacy and public health: a systematic review and integration of definitions and models. BMC Public Health 2012;12:80.

27 Pfaff H. The cologne patient questionnaire. Development and validation of a questionnaire of the involvement of patients as cotherapist: [Der Kölner Patientenfragebogen (KPF): Entwicklung und Validierung eines Fragebogens zur Erfassung der Einbindung des Patienten als Kotherapeuten]. Sankt Augustin: Asgard Verlag, 2003.

28 Mehnert A, Müller D, Lehmann C. Die deutsche Version des NCCN Distress-Thermometers [The German version of the NCCN distress thermometer]. Zeitschrift für Psychiatrie, Psychologie und Psychotherapie 2006;54:213-23.

29 Wirtz MA, Röttele N, Morfeld M, et al. Handling missing data in the short Form-12 health survey (SF-12): concordance of real patient data and data estimated by missing data imputation procedures. Assessment 2020:107319112095288.

30 Fauser D, Bethge M. [Propensity Score Methods to Estimate Treatment Effects: An Opportunity for Rehabilitation Service Research]. Rehabilitation 2019;58:50-8.

31 Cohen J. Statistical power analysis for the behavioral sciences. Burlington: Elsevier Science, 2013.

32 Livaudais JC, Franco R, Fei K, et al. Breast cancer treatment decision-making: are we asking too much of patients? J Gen Intern Med 2013;28:630-6. 